\title{
Über blutzuckersenkende Substanzen, deren chemische Struktur dem Guanidin nahe verwandt ist.
}

\author{
Von \\ Yoshio Shikinami, Shunyichi Yonechi, \\ (政 波戔雄) (米地俊一) \\ Shin-ichi Kawai und Tatsuo Hosono. \\ (河合或一) (細 野 辰雄) \\ (Aus der medizinischen Klinik ron Prof. Dr. T. Kumagai und \\ dem chemischen Institut ron Prof. Dr. R. Majima \\ an der Tohoku-Universitat zu Sendai.)
}

Es ist von T. Kumagai, Y. Shikinami und S. Kawai $\mathbf{~}^{\mathbf{1}}$ berichtet worden, dass bei verschiedenen Guanidinderivaten die blutzuckersenkende Wirkung Hand in Hand mit der Verlïngerung der Methylenkette zunimmt, während ihre Giftigkeit sich vermindert. Anschliessend an diese Arbeit haben wir Versuche mit einigen anderen organischen Verbindungen angestellt, die wir im folgenden mitteilen wollen.

Thioharnstoff. Wie man aus Tabelle I sieht, wirkt diese Substanz viel weniger blutzuckersenkend als Guanidin. Die Injektion von $0,4 \mathrm{~g}$ Thioharnstoff pro $\mathrm{kg}$ bei einem $1,2 \mathrm{~kg}$ wiegenden Kaninchen setzt den Blutzucker von $0,094 \%$ nur auf $0,073 \%$ herab. Eine grössere Menge verursacht dagegen Hyperglykämie. Bei einem Kaninchen, dem $4 \mathrm{~g}$ Thioharnstoff pro kg eingespritzt wurde, erhöhte sich z. B. der Blutzucker von 0,137\% auf $0,346 \%$, und das Tier verschied unter dem Bild sch l a f fer I Iäh mung.

Eine Reihe von Derivaten des Pseudothioharnstoffs, dessen Struktur dem Thioharnstoff ähnlich ist, kommt nun in Betracht.

Methylpsendothioharnstoff, bei dem eine Methylgruppe an den Schwefel des Psendothioharnstoffs gebunden ist, bringt in einer Menge von 50100-300 mg pro kg Körpergewicht relativ starke Hypoglykïmie hervor. Wir haben danach wie bei den Guanidinderivaten durch Verlängerung der

1) Kumagai, Kawai und Shikinami, Proc. Imp. Academy, 1928, 4, 23; K u ma* ga i, Shikinami and Hosono, Scientific Papers of the Institute of Physical and Chemical Research, 1928, 9, 27 I. 


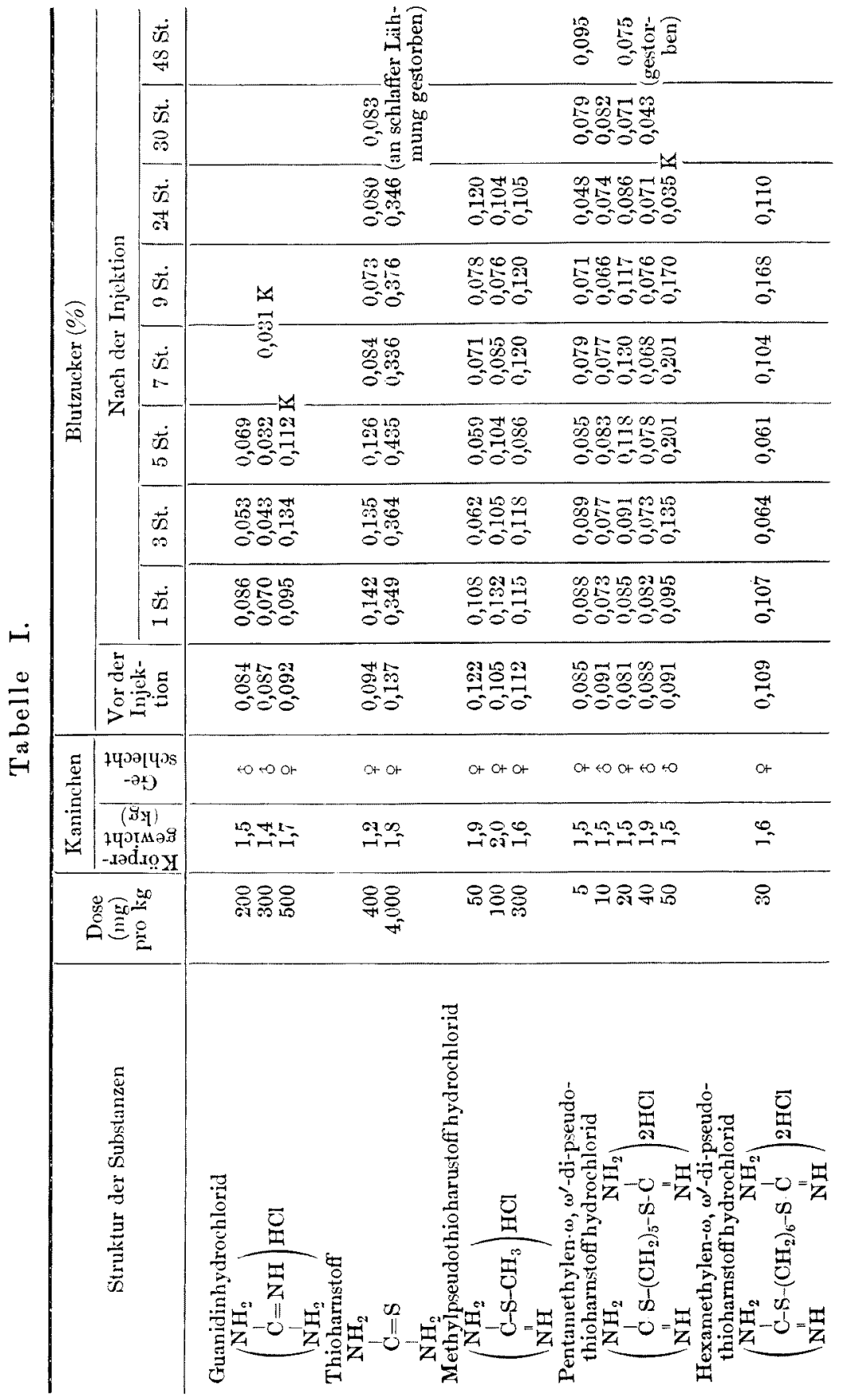




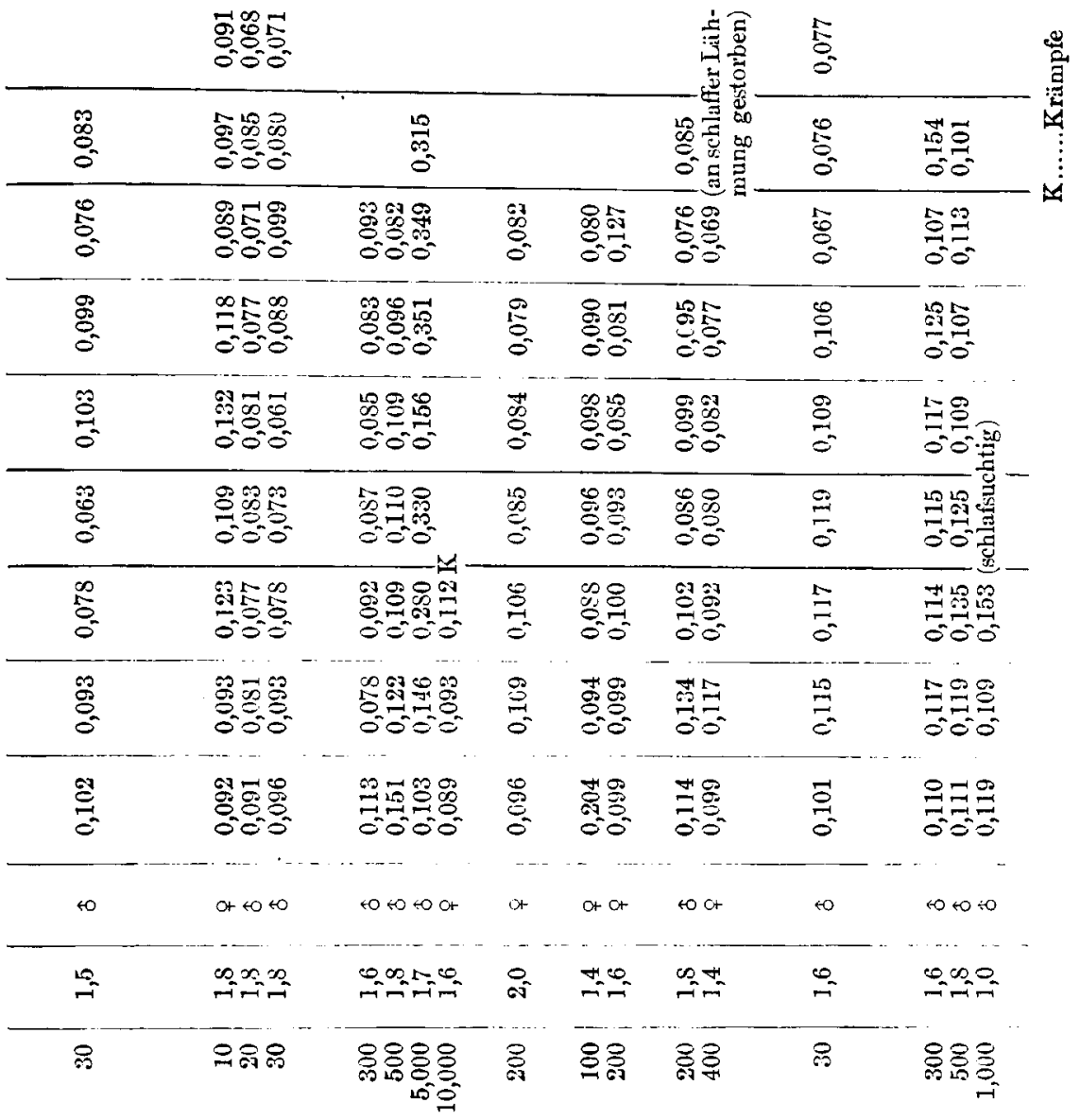

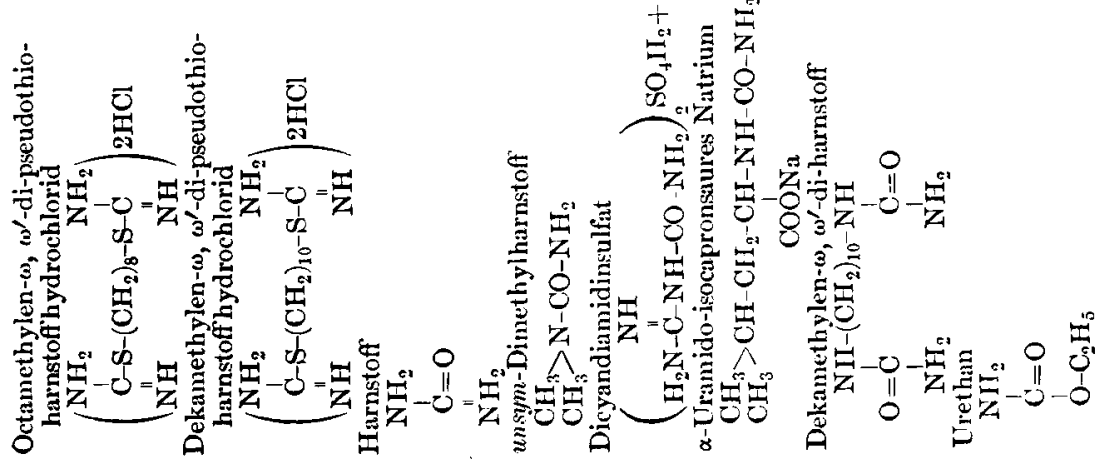


Methylenkette verschiedene Derivate dieser Verbindung synthetisch dargestellt und deren Wirkung untersucht. Pentamethylen- $\omega, \omega^{\prime}$-di-pseudothioharnstoff setzte in einer Menge von $5 \mathrm{mg}$ pro $\mathrm{kg}$ den Blutzucker des Kaninchens von $0,085 \%$ auf $0,048 \%$ herab und führte in einer Dase von $40 \mathrm{mg}$ bei einem anderen Kaninchen den Tod durch hypoglykämische Krümpfe herbei. Beim Hexa-, Okta- und Dekamethylen- $\omega, \omega^{\prime}$-di-pseudothioharnstoff ist der Einfluss auf den Blutzucker nicht wesentlich stärker als beim Pentamethylen- $\omega, \omega^{\prime}$-di-pseudothioharnstoff. Erst durch $30 \mathrm{mg}$ dieser Substanzen sinkt der Blutzuckerwert etwa um die Hälfte.

Urethan, dessen Struktur dem Guanidin auch ähnlich ist, wirkt gar nicht blutzuckersenkend, obwohl seine narkotische Wirkung mit steigender Dose zunimmt.

Harnstoff ist bekanntlich ein harntreibendes Mittel. Über seine blutzuckersenkende Wirkung liegen jedoch bis heute noch keine Berichte vor. Da die chemische Struktur des Harnstoffs dem Guanidin sehr ähnlich ist, fragten wir uns, ob er auch hypoglykïmisch wirkt. In der 'Tat bringt Harnstoff in einer Menge von 0,3-0,4 g pro kg etwa $30 \%$ ige Blutzuckersenkung hervor. Durch Anwendung einer grösseren Menge (5-10 g) geht indessen das Tier im Zustand der Blutzuckererhöhung unter Krärmpfen zugrunde. Nach Gigon ${ }^{2)}$ soll Harnstoff Hyperglykümie verursachen, aber seine Versuche wurden nur mit grossen Dosen ausgeführt und nicht mit kleineren Mengen, wie wir sie anwendeten.

Dicyandiamidin, das eine Verbindung des Harnstoffs mit dem Guanidin darstellt, übt in einer Menge von $0,2 \mathrm{~g}$ pro $\mathrm{kg}$ keine Wirkung auf den Blutzucker aus. Dagegen wirkt unsym.-Dimethylharustoff, der in seinem Molekül zwei Methylgruppen besitzt, in einer Menge von $0,2 \mathrm{~g}$ pro kg eingespritzt, schwach blutzuckersenkend.

Durch weitere Verlängerung der Methylenkette dargestelltes $\%$-Uramidoisocapronsaures Natrium wirkt in einer Menge von $0,2 \mathrm{~g}$ pro $\mathrm{kg}$ sch wach, bei $0,4 \mathrm{~g}$ deutlich blutzuckersenkend. Bei Anwendung von $0,4 \mathrm{~g}$ pro $\mathrm{kg}$ sank nïmlich der Blutzucker von $0,099 \%$ auf $0,069 \%$, und das Tier ging unter dem Bild schlaffer Lühmung zugrunde.

Dekamethylen- $\omega, \omega^{\prime}$-di-harnstoff ist kaum wasserlöslich. Eine Lösung davon in warmem Alkohol, und zwar $30 \mathrm{mg}$ Substanz pro kg eingespritzt, setzte den Blutzucker von 0,101\% auf 0,067\% herab. (Tabelle I)

Da Harnstoff unter den erwähnten Verbindungen verhältnismässig wenig giftig ist, wollten wir nun die Art und Weise seiner blutzuckersen-

2) Gigon, Schweiz. med. Wochenschr., 1927, 294. 


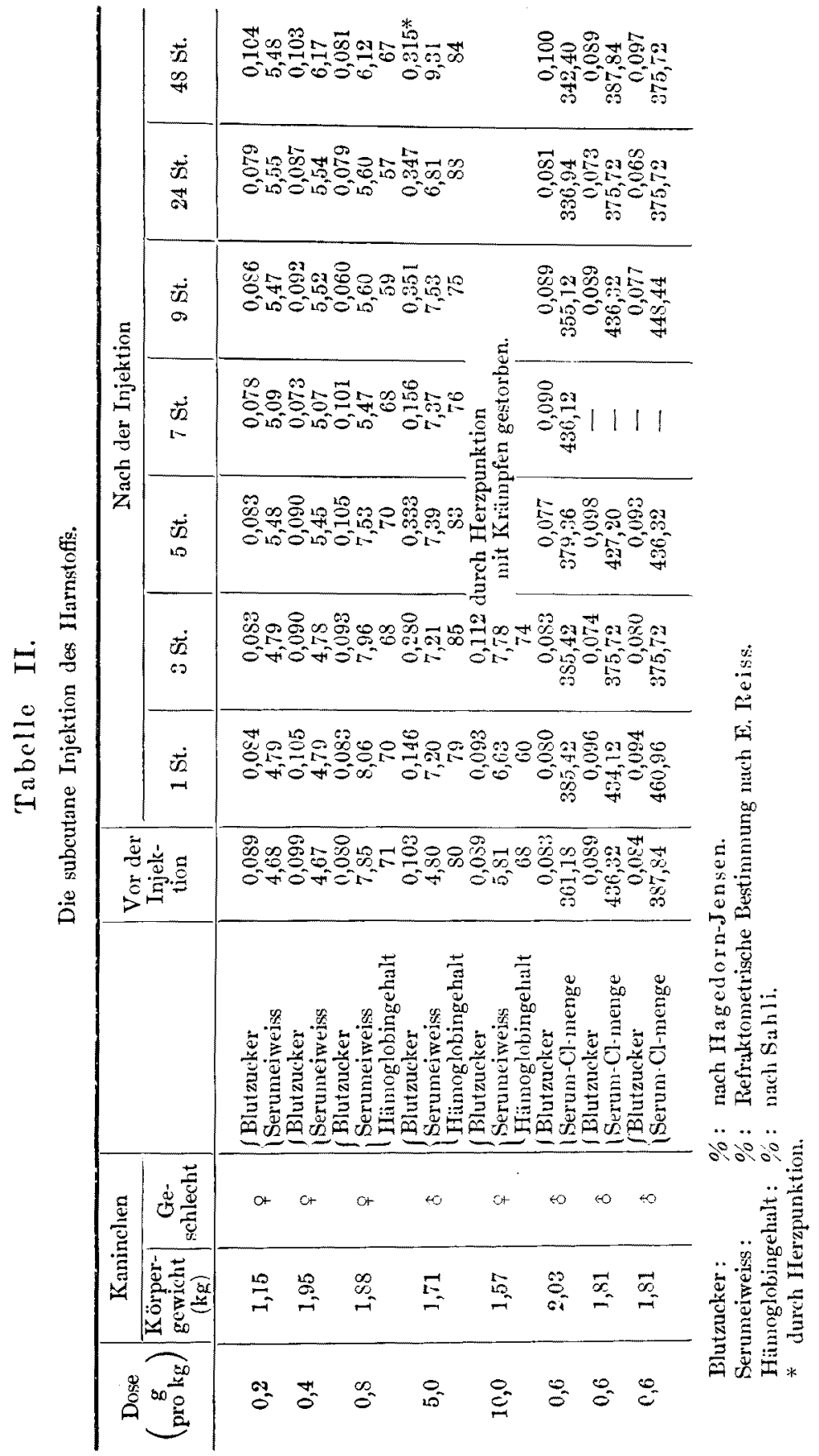


kenden Wirkung erkennen. Man muss zuerst sehen, ob die Erniedrigung des Blutzuckers infolge Harnstoffs durch die Konzentrationsäuderung des Bluts bedingt ist oder nicht, da ja Harnstoff als Diureticum benutzt wird. Nun ergab sich durch die Bestimmung des Hämoglobingehalts, des Serumeiweisses und der Serumchlormenge, dass perorale sowie parenterale Zufuhr von etwa $0,4 \mathrm{~g}$ Harustoff pro kg ungeführ 10\% ige Hydrümie zustandebringt, während der Blutzucker sich sogar um $30 \%$ vermindert. Dieses Ergebnis deutet darauf hin, dass die Hypoglykämie durch Harnstoff nicht als Folge der Hydrämie auftritt. (Tabelle II)

Perorale Gabe von Harnstoff bei Diabetikern in einer Menge von $0,6 \mathrm{~g}$ pro $\mathrm{kg}$ übt zwar auf den Blutzucker keinen nachweisbaren Einfluss aus, aber die Glykosurie kann dadurch deutlich gehemmt werden. In der folgenden Figur zeigen wir einen diesbezüglichen Versuch bei cinem Diabetiker, der wïhrend der ganzen Versuchsperiode täglich 120,2 g Eiweiss, $162,5 \mathrm{~g}$ Fett, $113,5 \mathrm{~g}$ Kohlehydrat (50 Kalorien pro kg) erhielt.

Pernale Anwendung des IIarnstoffes bei einem Diabetiker.

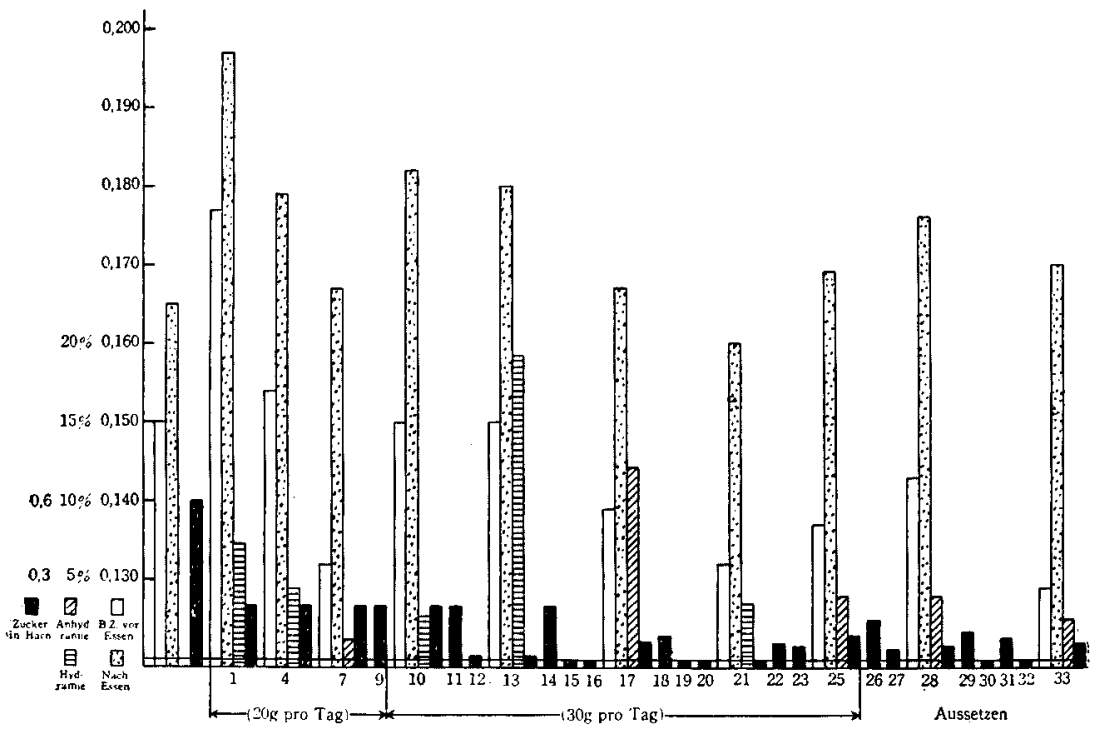

Dekamethylen- $\omega, \omega^{\prime}$-di-harnstoff, der durch Verlängerung der Methylenkette dargestellt wurde, ist kaum wasserlöslich, so dass seine Anwendung für unseren $Z$ weck schwierig ist. Ja, die Harnstoffderivate sind umso schwerer löslich, je lïnger ihre Methylenkette wird. 


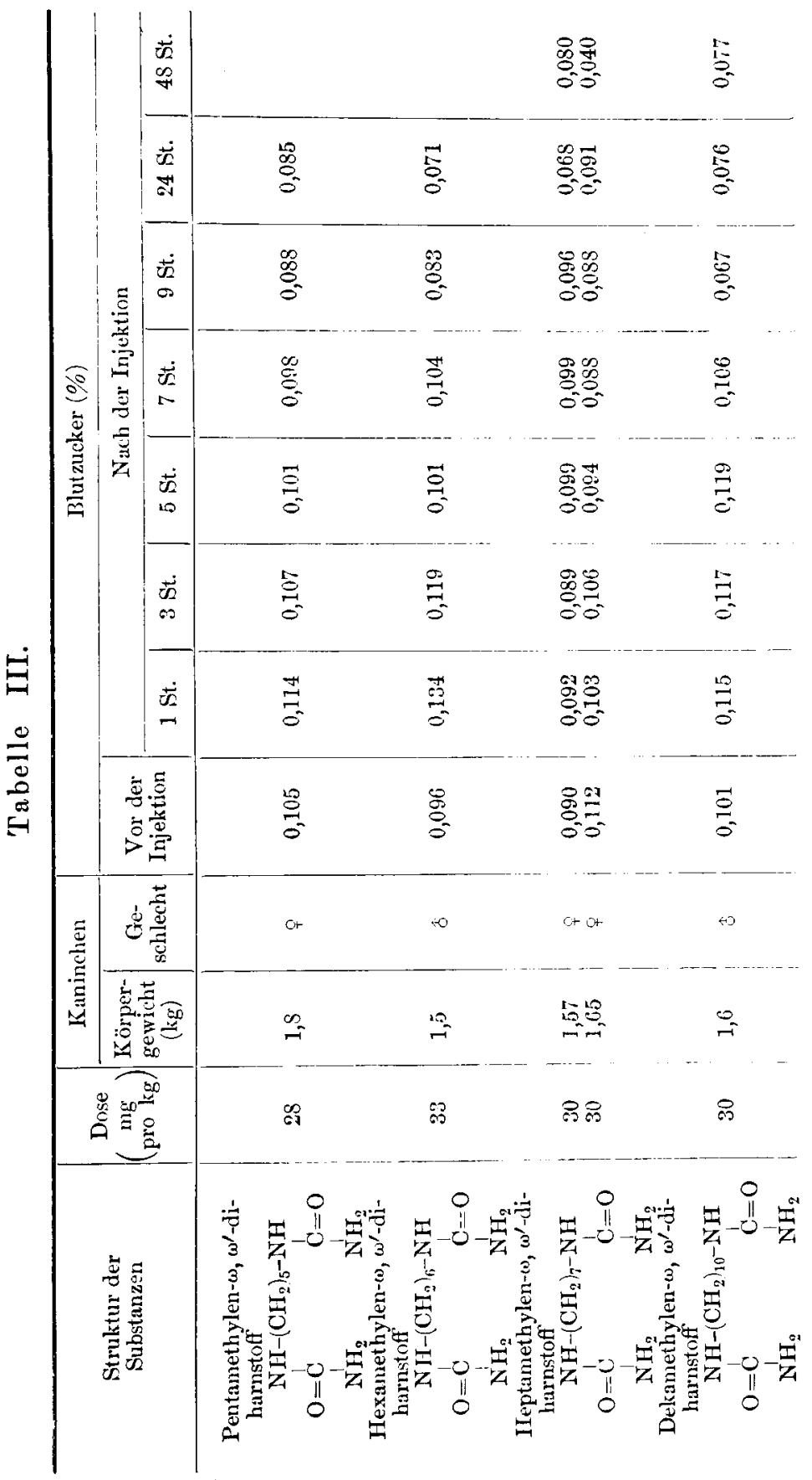




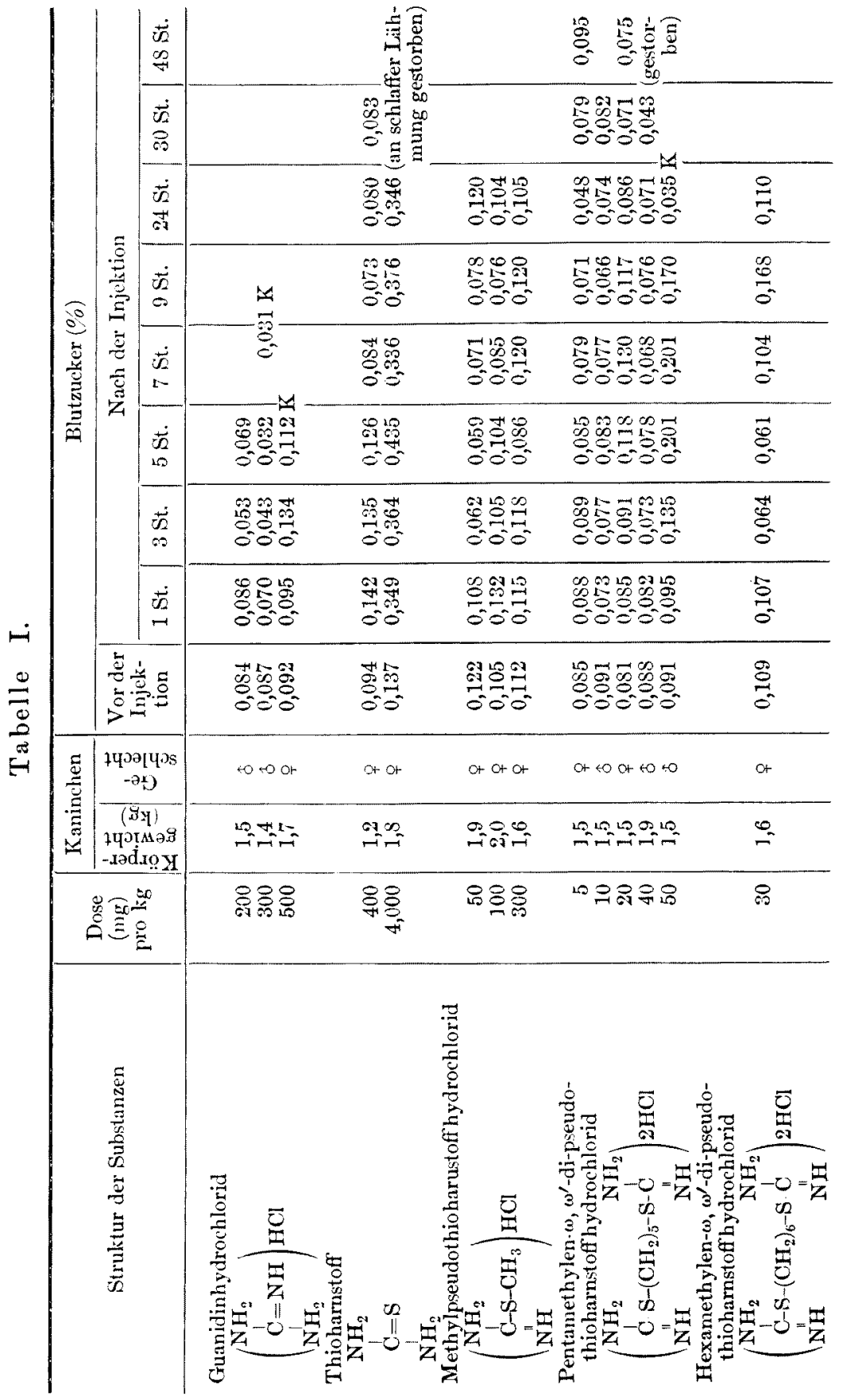




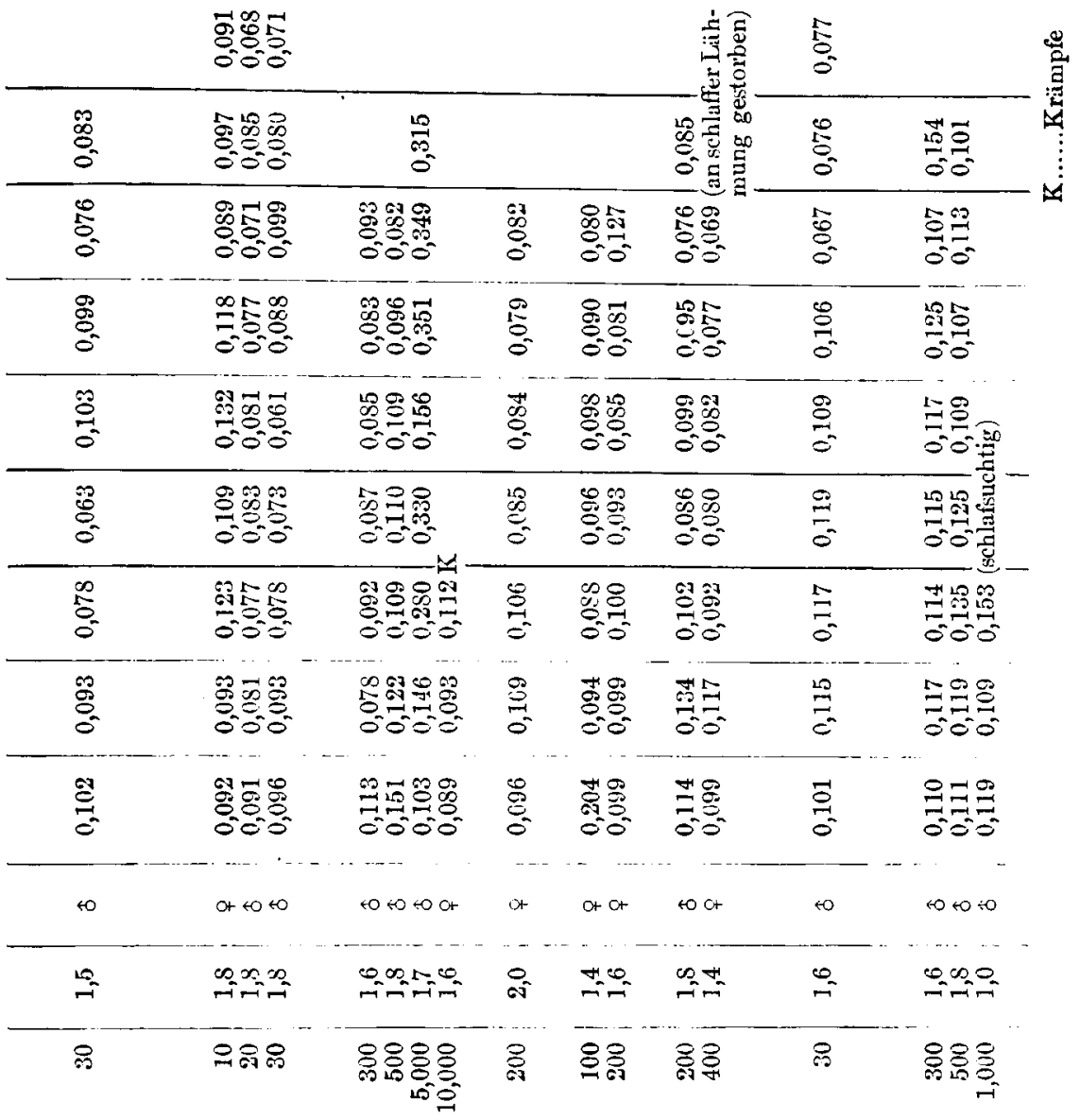

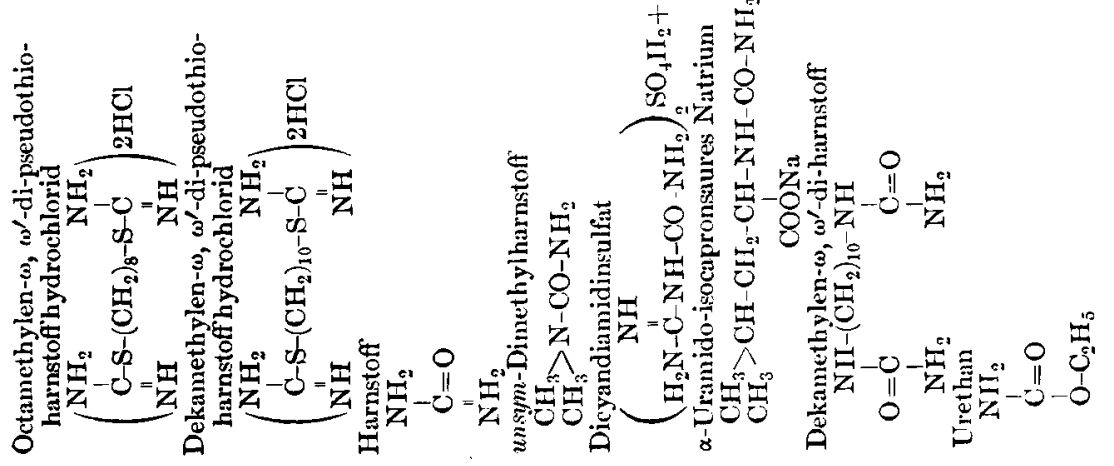




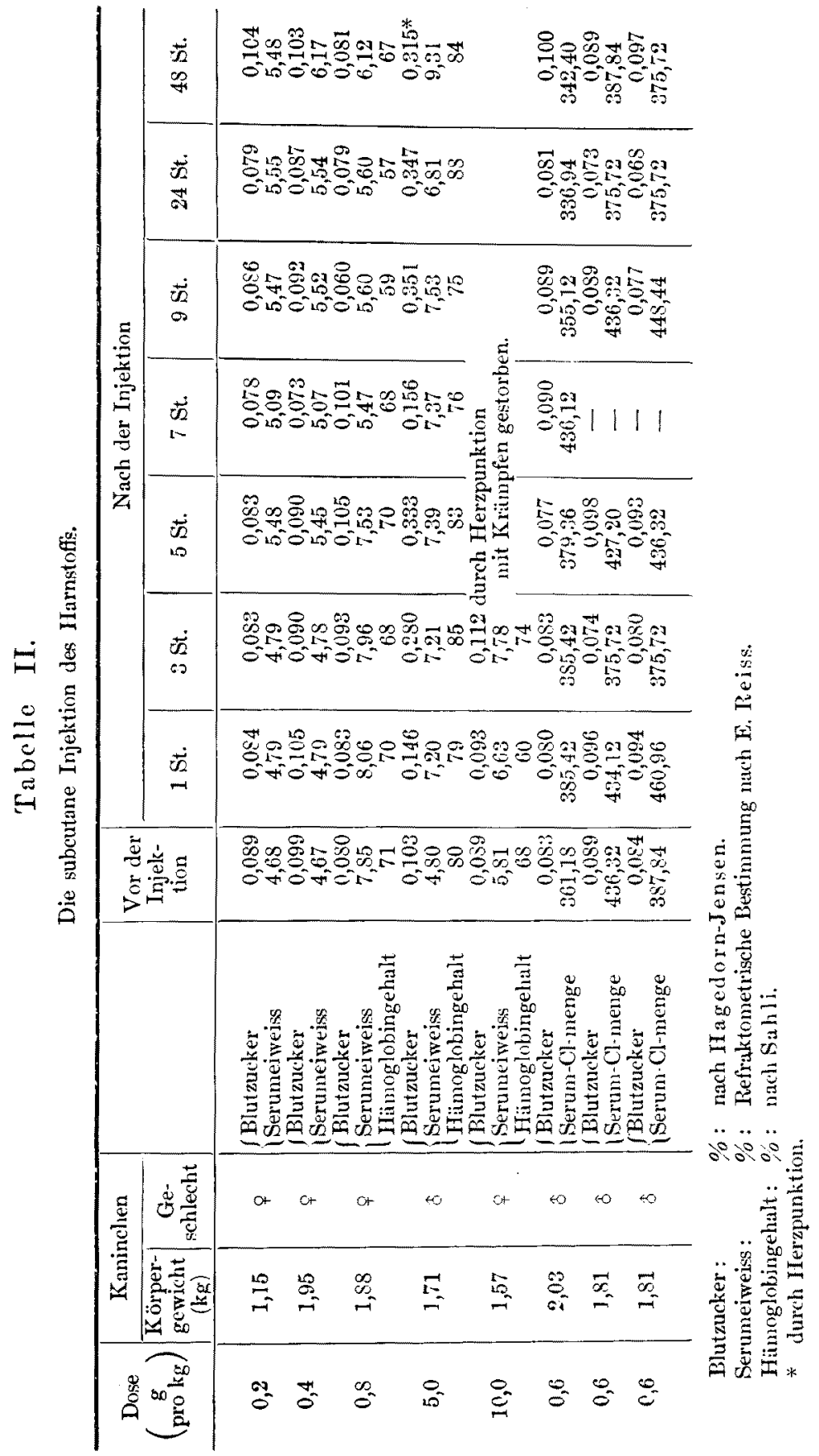




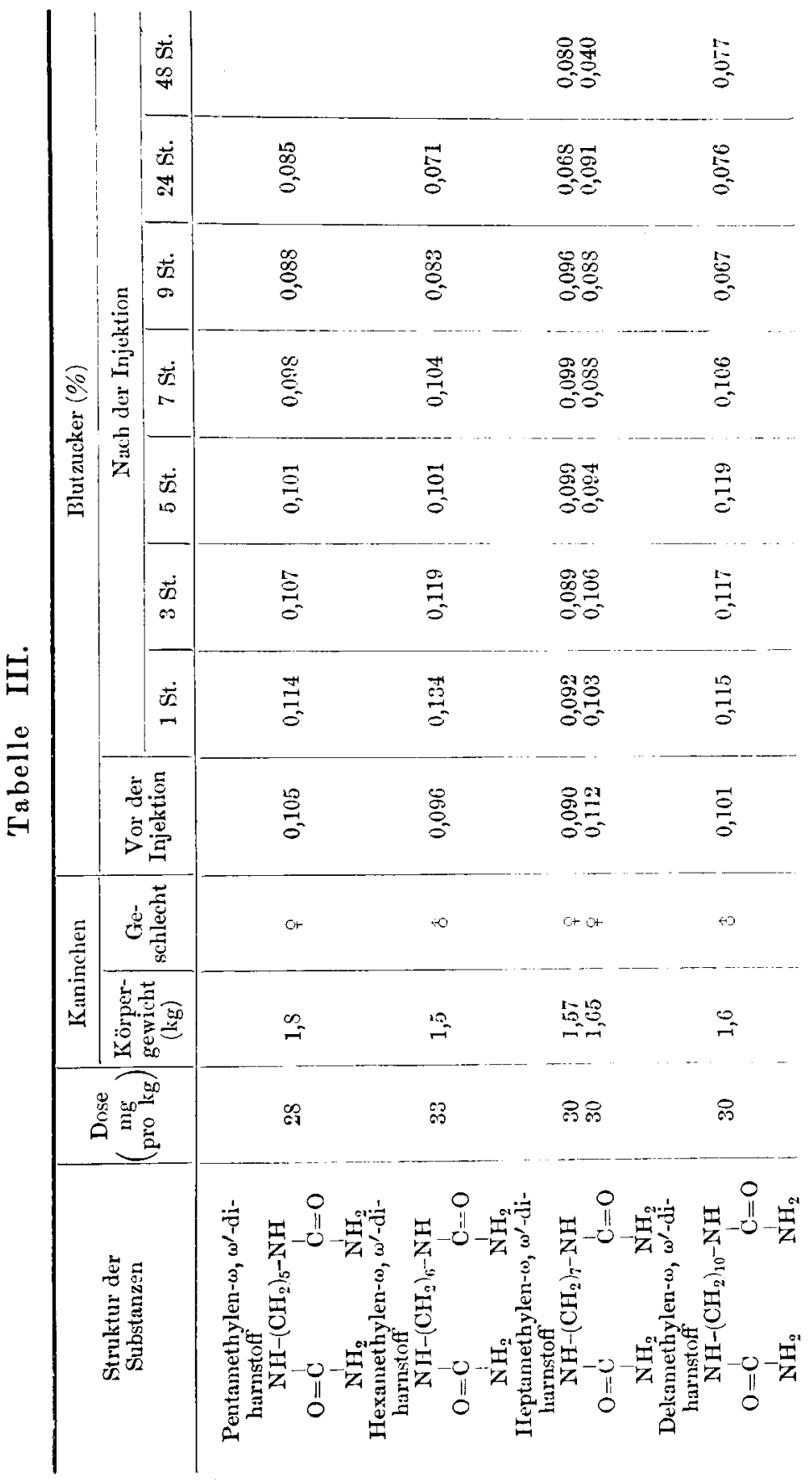


Unsere nüchste Aufgabe war also, unter deu Polymethylen- $\omega, \omega^{\prime}$-di-harnstoffen diejenigen auszuwählen, die wasserlöslich und zwar hypoglykümisch wirksam sind. Solche sind Penta-, Hexa- und Heptamethylen$\omega$, $\omega^{\prime}$-di-harnstoff. Der erste, der Petamethylen- $\omega, \omega^{\prime}$-di-harnstoff, ist zwar weit löslicher als der Dekamethylen- $\omega, \omega^{\prime}$-di-harnstoff, zeigt aber sogar bei $30 \mathrm{mg}$ pro $\mathrm{kg}$ fust keine blutzuckersenkonde Wirkung. Hexaund Heptamethylen- $\omega, \omega^{\prime}$-di-harnstoff sind weniger löslich als Pentamethylen- $\omega, \omega^{\prime}$-diharnstoff, aber im Vergleich mit dem Dekametliylendiharnstoff löslicher, und $30 \mathrm{mg}$ jeder dieser Substanzen ruft ziemlich starke Hypoglykämie hervor. Besonders beim Heptamethylen- $\omega$, $\omega^{\prime}$-di-harnstoff steht die Wirkung der des Dekamethylen- $\omega$, $\omega^{\prime}$-diharnstoffs nicht nach. Deshalb wollen wir nun fortau den Hexa- und Heptamethylen- $\omega$, $\iota o^{\prime}$-di-harnstoff klinisch probieren. (Tabelle III)

Ausserdem sind noch einige Amidinderivate untersucht worden. Diese sind in Gegensatz zu den Harnstoffderivaten leicht wasserlöslich. Die blutzuckersenkende Wirkung tritt erst in Derivat mit sechs Methylenketten auf. Die mit 7, 8 und 10 Methylenketten haben auch gleich starke Wirkung. (Tabelle IV)

Batrachtet man die Mitteilung von $\mathrm{T}$. Kumagai, Y. Shikinami und S. Kawai über Guanidinderivate und unsere Untersuchungen über Pseudothioharnstoff-, Harnstoff- und Amidinderivate, so ergibt sich daraus, dass ausser Verbindungen mit freien Guanidinmolekülen anch solche mit freien Aminogruppen hypoglykämisch wirksam sind, und zwar tritt diese Wirkung umso

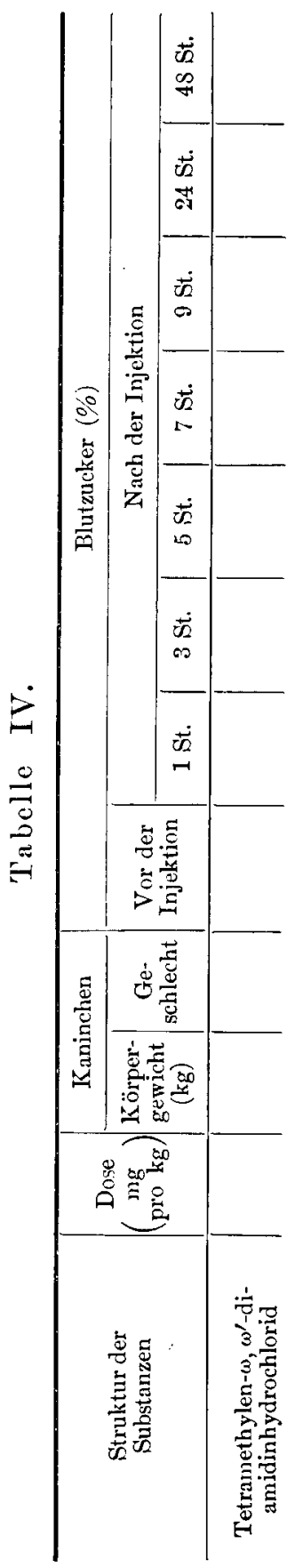




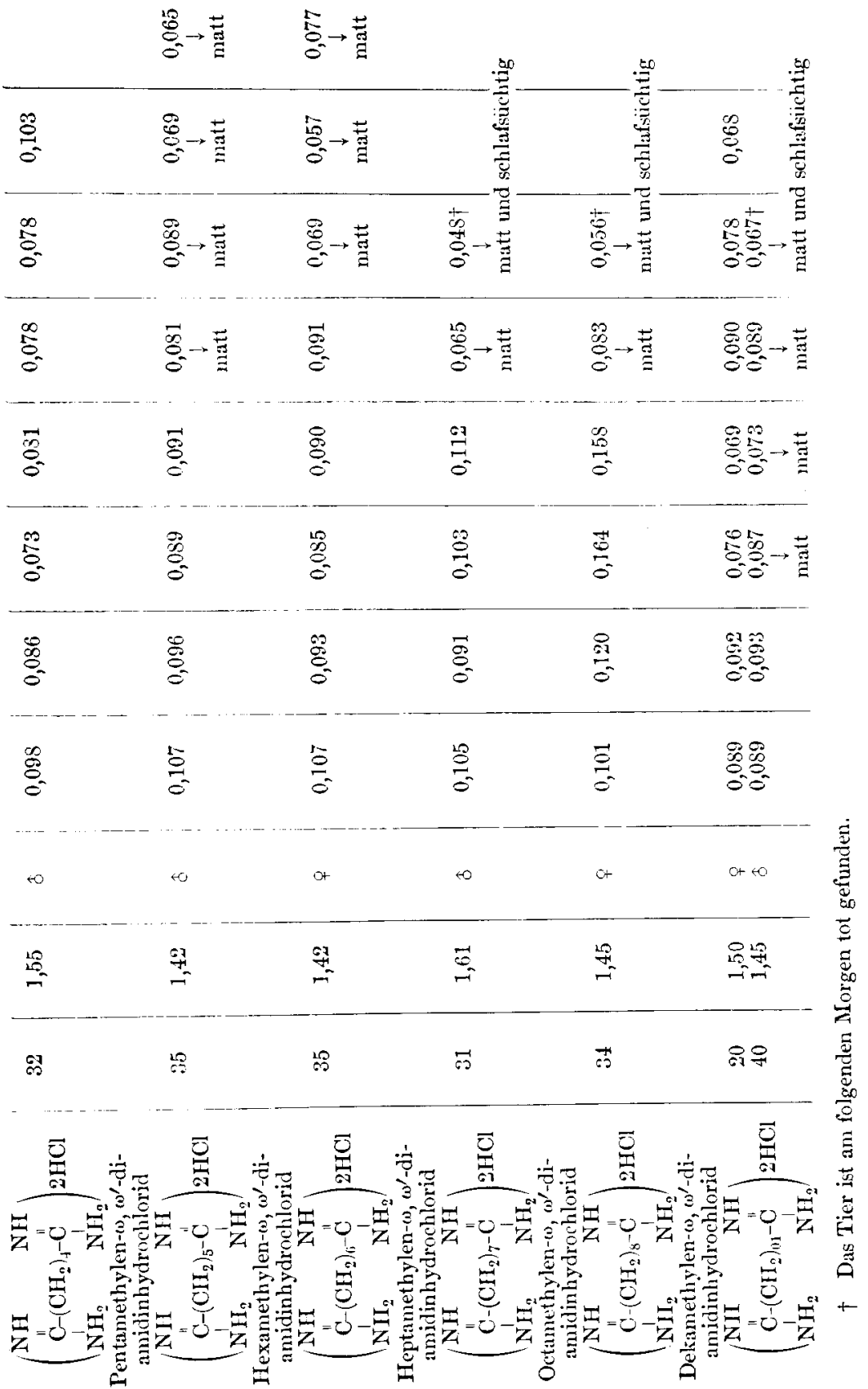


stïrker zutage, je länger die Methylenkette wird. Ausserdem hat man noch den Eindruck, dass solche Derivate mit ungerader Zahl der Methylenketten stïrker wirken als die mit gerader Zahl. 